\title{
Implementation of conifer somatic embryogenesis in clonal forestry: technical requirements and deployment considerations
}

\author{
Yill-Sung Park* \\ Natural Resources Canada, Canadian Forest Service, Atlantic Forestry Centre, PO Box 4000, Fredericton, New Brunswick E3B 5P7, Canada
}

(Received 16 August 2001; accepted 16 January 2002)

\begin{abstract}
Cloning of trees using somatic embryogenesis (SE) could have a major impact on tree breeding and commercial plantation forestry. In conjunction with cryopreservation, SE offers an opportunity to develop highly valuable clone lines. Commercial deployment of such genetically tested clone lines in forestry will dramatically increase forest productivity over any available conventional tree improvement techniques. However, sufficient technical advances must be made to use SE in clonal forestry. Progress in SE techniques and genetic stability of clones is reviewed, using white spruce (Picea glauca) and eastern white pine (Pinus strobus) as model species. There are several other issues in implementing clonal forestry. The main concern is managing clonal plantations for optimal genetic gain and diversity. The issues and considerations for selecting appropriate numbers of clones and deployment strategies are discussed. A clonal deployment strategy using a "mixture of clones and seedlings" is proposed for eastern Canada.
\end{abstract}

somatic embryogenesis / cryopreservation / genetic stability / clonal forestry / tree improvement

Résumé - Mise en œuvre de l'embryogenèse somatique des conifères en foresterie multiclonale : exigences techniques et considérations sur son utilisation. Le clonage des arbres réalisé à l'aide de l'embryogenèse somatique (ES) pourrait avoir un impact majeur sur l'amélioration des arbres et la foresterie commerciale de plantation. L'ES, utilisée de pair avec la cryoconservation, offre la possibilité de développer des lignées multiclonales de grande valeur. L'utilisation commerciale de telles lignées multiclonales testées génétiquement permettra d'augmenter de façon radicale la productivité forestière comparativement aux autres techniques d'amélioration génétique classique. L'utilisation de l'ES en foresterie multiclonale exige toutefois certaines avancées techniques. Les progrès réalisés au niveau des techniques d'ES et de la stabilité des clones sont présentés en utilisant comme espèces modèles l'épinette blanche (Picea glauca) et le pin blanc (Pinus strobus). De plus, lors de l'instauration d'un programme de foresterie multiclonale, il y a de nombreux obstacles à surmonter. Le principal point à surveiller est l'aménagement des plantations multiclonales afin de maximiser à la fois le gain et la diversité génétiques. Les problèmes rencontrés et les considérations à prendre en compte lors du choix du nombre approprié de clones et de la stratégie de déploiement sont également discutés. Une stratégie de déploiement multiclonal basée sur le recours à un mélange de clones et de semis est proposée pour l'est du Canada.

embryogenèse somatique / cryoconservation / stabilité génétique / foresterie multiclonale / amélioration des arbres

\section{INTRODUCTION}

Clonal forestry may be broadly defined as any use of clonally propagated trees in forestry, including the use of bulk-propagated families. However, more restrictively, it refers to the use of only tested clones in plantation forestry. Clonal forestry has been practised with some hardwood species, such as poplar. Recently, however, the opportunities for clonal forestry with conifer species have generated keen interest $[5,16]$. This is due primarily to advances in traditional as well as in in vitro vegetative propagation techniques.

There are many advantages to practising clonal forestry, including (1) consistent production of the same genotypes over time, (2) capture of larger genetic gains than possible with any conventional tree breeding technique, (3) flexibility to rapidly deploy suitable clones given changing breeding goals and/or environmental conditions, and (4) ability to manage genetic diversity and genetic gain in plantation for-

* Correspondence and reprints

Tel.: (506) 452 3585; fax: (506) 452 3525; e-mail: ypark@ nrcan.gc.ca 
estry [14]. Despite these advantages, clonal forestry has rarely been practised with conifers, mainly because of the general lack of an efficient clonal propagation system that can mass produce genetically tested material.

To practise clonal forestry, an effective mass vegetative propagation method must be available. The traditional vegetative propagation technique is rooting of cuttings. This method has been particularly effective and cost efficient for hardwood species, such as poplar. In conifer species, however, mass propagation of true-to-type trees by rooted cuttings is generally possible only with seedlings up to about 5 years of age. This is a serious limitation because, by the time the genetic superiority of a clone has been determined through lengthy genetic tests, the donor plant has become too old for further mass propagation by rooting of cuttings.

Recent advances in somatic embryogenesis (SE) technology make it possible to circumvent this problem, at least for some conifer species. For most spruce and a few larch and pine species, about $60 \%$ of the seeds will produce embryogenic cultures and, of these, about $80 \%$ will form clonal plants suitable for planting in the field. These rates are high enough for industrial application, particularly for developing high-value clonal varieties.

The most important advantage of conifer propagation by $\mathrm{SE}$ is that the embryogenic clonal lines can be cryopreserved in liquid nitrogen, while corresponding trees are tested in the field. This provides an opportunity to develop "clonal varieties" by thawing and re-propagating cryopreserved ET clones that have shown genetic superiority in the field tests. In the past, the development of forest "tree varieties" in conifers was impractical because we were unable to produce the same genotypes consistently over time. By repeating cycles of cryopreservation, thawing, proliferation, and re-cryopreservation, sufficient quantities of tested ET clones can be maintained indefinitely in cryogenic storage. This offers great flexibility to propagate desired genotypes consistently at any time.

The purposes of this paper are (1) to examine the technical requirements for conifer SE as they relate to its use in clonal forestry, and (2) to discuss the issues in developing clonal deployment strategies. This presentation is based on a review of our experience with spruce and pine species in eastern Canada.

\section{IMPLEMENTATION OF SOMATIC EMBRYOGENESIS IN TREE BREEDING PROGRAMS}

Most tree breeding programs have adopted a system of recurrent selection to obtain successive generations of breeding material. In each generation, selected materials are bred to obtain a high level of genetic gain, while maintaining genetic diversity in the breeding population. Conventionally, the selected materials are also planted in seed orchards to produce

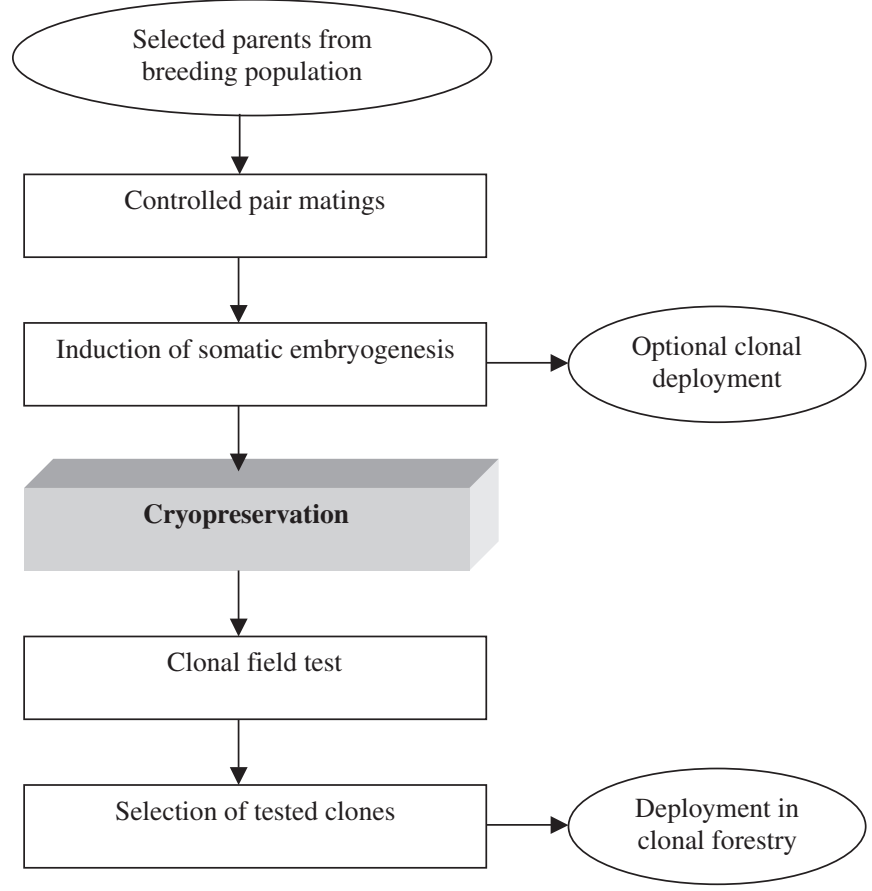

Figure 1. A simplified flowchart for clonal deployment strategy using somatic embryogenesis and cryopreservation. Modified from [13].

genetically improved seeds. Although traditional seed orchards provide genetically improved seeds, breeding strategies using vegetative propagation have additional advantages. SE can be such a technology that would make the practice of clonal forestry possible.

A simplified implementation of SE in tree breeding is illustrated in figure 1. In the context of advanced-generation breeding, the implementation strategy could begin with a set of selected parents from a breeding population maintained in a breeding garden or breeding greenhouse. Controlled crossings are made between pairs of these parents. Small quantities of high quality, full-sib seeds, resulting from these crosses, are then used to initiate SE. Once embryogenic tissue (ET) is initiated, it is proliferated and clonal ET lines are cryopreserved. After a few weeks, a portion of ET for each clone is thawed and propagated to produce a small number of plants using the normal SE process. The plants from these clones are then planted in replicated clonal field tests. The performance of clones is assessed at regular intervals until the trees reach rotation age, thus accumulating genetic information continuously as genetic testing progresses. Once field tests have identified the best performing clones, the corresponding ET lines are thawed from cryopreservation and used to produce planting stock for deployment in clonal forestry. Genetic testing in the field is an important part of a clonal forestry scheme for initially identifying suitable clones to use in clonal mixtures. Genetic information obtained at an early age can be used to select appropriate clones; 
however, the most useful data is obtained at rotation age. Thus, the test plantation provides updated information continuously to refine selection of clones throughout the rotation age.

In this strategy, there are two opportunities to deploy vegetative propagagules. The first opportunity is to deploy cloned trees of controlled crossed families before field testing. As the parents used in the controlled crosses represent the best individuals in the previous generation, the genetic gain is roughly the same as that from the next generation clonal seed orchards. However, genetic efficiency is much greater in clonal planting stock because certain inefficiencies of seed orchards, such as pollen contamination and asynchronous flowering, have been avoided by making controlled crosses. Although SE can be used for this type of clonal deployment, rooting of cuttings may be used more effectively than SE. As described previously, the real opportunity with SE is to select best-tested clones and deploy them in clonal forestry. Although a testing period is required to identify best performing clones, the genetic gain from this scheme is much greater than untested deployment schemes. For example, genetic gains in 5-year height from seed orchards of Picea mariana and Pinus banksiana were 6 and 5\%, respectively (Tosh, New Brunswick Tree Improvement Coucil, pers. comm.) but, in a 5-year-old clonal test of Picea glauca, the ten best of 300 clones had a $45 \%$ height advantage over the average of all clones in the test (unpublished data).

\section{TECHNICAL ISSUES OF CONIFER SOMATIC EMBRYOGENESIS}

The clonal deployment strategy using tested clones depends entirely on developing embryogenic clone lines and cryogenically storing them during field testing. For successful implementation of SE in clonal forestry, the technique has to be sufficiently refined, i.e., high initiation and plant conversion rates and maintenance of genetic stability/integrity during cryopreservation. Automated somatic embryo handling systems, such as artificial seed or encapsulated somatic embryos, would greatly enhance SE implementation in tree breeding programs.

\subsection{Initiation and plant conversion rates}

Sufficiently high SE initiation and subsequent plant conversion rates are important to maintain genetic diversity of clonal plantations while achieving a high level of genetic gain. Improving induction rate has been a major area of SE research and is influenced by several factors, such as tissue culture media, stage of maturity in zygotic embryo (ZE), and genetic influence.

The current SE initiation rate in spruce species, including P. glauca, P. mariana, and Picea abies, is sufficiently high, at over $65 \%$, when immature ZE explants are used. Of these, about $80 \%$ of embryogenic clones produce plants. The initia- tion rate in pine species had been low. However, we recently achieved a high SE initiation rate in Pinus strobus by manipulating plant growth regulators [7]. There seem to be opportunities for improving SE initiation in semi-recalcitrant and recalcitrant species by manipulating initiation media, but there are large differences in SE initiation among species. Modification and refinement of initiation media continues to be an important aspect of SE research, because an efficient SE protocol is not yet available for many conifer species.

The maturation stage of ZE is critically important, especially for pine species. In $P$. strobus, for example, the most responsive stage of ZE development is immediately following fertilization, i.e., pre- and post-cleavage stage before the appearance of a dominant embryo. This is evidenced by a sharp increase in induction rate during the anticipated fertilization period and subsequent gradual decline as ZE development continues (figure 2) [7]. Careful monitoring of the ZE development stage may result in high initiation rates.

Availability of immature ZEs at the most responsive stage is often limited. To extend the availability of these ZEs, two cold storage experiments were conducted using 20 open-pollinated families of $P$. strobus. The first experiment involved storing cones at $-3{ }^{\circ} \mathrm{C}$. The cones were retrieved weekly and subjected to an SE initiation treatment. As expected, the percentage of initiation declined as the length of the frozen storage period increased. The percentages of SE initiation for fresh ZE explants and those held in frozen storage for 14, 21, 28,35 , and 42 days were $43.9,26.3,11.2,7.3,5.8$, and $0.6 \%$,

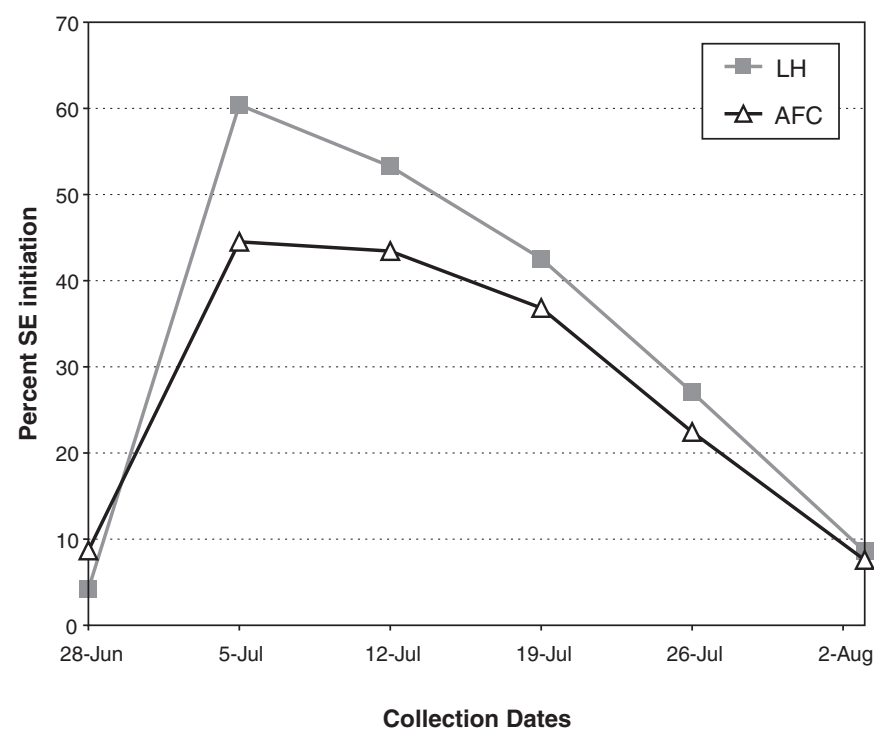

Figure 2. Percent SE induction in $P$. strobus on two initiation media during zygotic embryo development in 1999. LH is optimized initiation protocol based on Litvay medium and AFC is initiation medium formulated in our laboratory with different base elements than LH medium. Both media had the same optimized levels of growth regulators [7]. 
respectively. Significant family effects were also found, indicating genetic influence.

The second experiment involved storing of cones in a refrigerator at $3{ }^{\circ} \mathrm{C}$, followed by initiation treatment. The percentages of SE initiation after $0,7,21,40$, and 100 days were $49.7,51.5,51.2,50.1$, and $38.8 \%$, respectively. These results indicate that eastern white pine cones may be stored in a refrigerator for at least 40 days without reducing embryogenic capacity. In some cases, refrigeration even stimulated SE induction.

The genetic influence during the SE process is well known. Understanding genetic control is an important element in improving the SE process. Depending on the type and magnitude of genetic variation, improved SE initiation may be introduced in recalcitrant genotypes. Based on the quantitative genetic analysis of 30 full-sib families derived by diallel crossing, we were able to partition the total genetic variation into separate genetic components [11, 12]. As illustrated in figure 3, the initiation phase of SE was under strong genetic control (69\% of total variance was due to genetic effects), but the genetic influence declined steadily through proliferation (38\%), maturation (9\%), and germination phases (3\%). This indicates that it is the initiation phase of SE that can be manipulated most effectively by breeding because a large amount of additive variance exists, accounting for

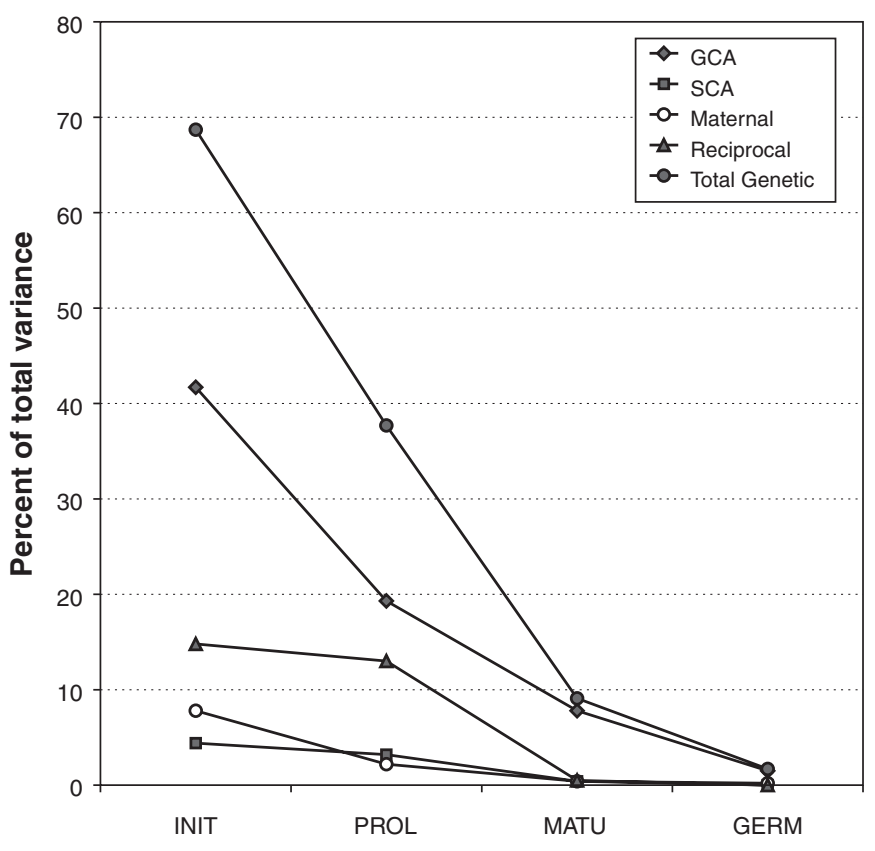

Phases of SE

Figure 3. Changes in genetic variance components during initiation (INIT), proliferation (PROL), maturation (MAT), and germination (GERM) phases of P. glauca SE. The legend, GCA, SCA, Maternal and Reciprocal indicate variance components due to general combining ability, specific combining ability, maternal and reciprocal effects, respectively [13].
$42 \%$ of total variance. Only limited improvements in maturation and germination can be obtained by breeding, however. There was no correlation among the different phases of SE. There was also a significant genetic influence in initiation of SE in P. strobus [7].

\subsection{Genetic stability of cryopreserved clones}

The clonal forestry strategy discussed earlier hinges on cryopreservation. In general, cryopreservation of ET is accomplished easily [1]. However, it is important to demonstrate that embryogenic clones are maintained without change in genetic makeup or loss of viability during cryogenic storage. To determine this, a set of 12 clones of $P$. glauca was thawed at two dates, a year apart, i.e., after 3 and 4 years in cryopreservation. The clones retrieved from cryopreservation were propagated through the SE process, grown in a greenhouse, and planted in a field test. Comparisons of clones between the two thawing dates were carried out using in vitro SE traits (i.e., maturation and germination characteristics), ex vitro morphological traits (i.e., greenhouse growth characteristics), and molecular markers. Examination of in vitro and ex vitro traits produced highly consistent results between the two thawing dates, indicating that genetic integrity is maintained during cryogenic storage [13].

Genetic stability of six randomly selected clones was evaluated using randomly amplified polymorphic DNA (RAPD) fingerprints [2]. Variant banding patterns were detected in two clones' in vitro embryogenic cultures 12 months after they were reestablished following 3 years of cryopreservation. Variant banding patterns were also found from trees regenerated from aberrant somatic embryos, such as somatic embryos with cotyledon deficiency, precocious germination, or other abnormal shapes. There was no banding pattern variation among the plants of same clone regenerated from somatic embryos that were normal in appearance, regardless of thawing dates. These results suggest that it is important to avoid a prolonged sub-culture and to select somatic embryos of normal morphology when propagating.

The genetic integrity of clonal lines developed by SE needs attention, particularly in pines. In pines, megagametophytes commonly contain multiple archegonia, which are thus capable of producing multiple genotypes within a megagametophyte. As the megagametophytes are routinely used for SE initiation, there is a possibility that an ET clone may contain mixed genotypes. One means of "purifying" clone lines is through re-initiation of ET from mature somatic embryos. Re-initiation has been obtained from P. strobus and $P$. banksiana, but at a lower rate. Initiation of SE from mature zygotic embryos has also been achieved for $P$. strobus [3]. 


\subsection{Encapsulated somatic embryos and alternatives}

The development of efficient somatic embryo handling techniques, such as encapsulated embryos or artificial seeds, is highly desirable because stock production by SE, which involves picking and germinating somatic embryos and subsequent transplanting into containerized growing medium in a greenhouse, is very labor intensive and thus expensive. Eliminating or automating any of these manual steps would reduce the production cost. We conducted an experiment with somatic embryos encapsulated in calcium alginate as well as direct germination of somatic embryos in peat plugs. To date, we have mixed results, requiring further refinement.

However, because effective artificial seed technology is still lacking, mass vegetative multiplication of superior clones by serial rooting of cuttings can be used as an alternative. Once field testing has shown which are the best clones, corresponding clones are thawed from cryopresevation and propagated to produce a few juvenile plants by SE which are then used as donor (stock) plants. Mass production of stecklings by rooting of cuttings from juvenile stock plants is achieved easily, particularly for spruce species. These stock plants can produce rooted cuttings for about 5 years. The cost of producing stecklings from stock plants is about twice the cost of seedlings; 1 million stecklings are produced annually in New Brunswick (Adams, Irving, Ltd, pers. comm.).

\subsection{Clonal field testing}

The theory of genetic testing in tree improvement is a well-established discipline and is equally relevant to clonal testing of SE-derived trees. One aspect of field testing of clonally propagated trees is to determine genetic fidelity of trees relative to comparable seedlings [4], such as freedom from plagiotrophism, early maturation, and other abnormalities. To date, our SE-derived trees of P. glauca and $P$. mariana in the field test have shown no such abnormalities at age 9 .

The primary reason for conducting clonal tests is to identify suitable clones for deployment. Clonal testing generally involves a number of candidate clones evaluated over a range of common test sites with respect to traits of interest. Normally, testing a large number of clones will result in larger genetic gain than a small number. However, clonal testing is constrained by limitations in logistics and resources. Raising test plants of many clones with comparable growth conditions or qualities by the SE process poses a logistics problem because the SE procedure is labor intensive, requiring several distinct steps, i.e., initiation, maturation, germination, and transplantion into containers for greenhouse culture, which may require different timing. Nevertheless, it is important to establish replicated common garden clonal tests containing a large but manageable number of clones. Alternatively, the test plants can be produced by serial rooting of cuttings from a few donor plants of each clone.
Currently, in New Brunswick, the number of clones included in a clonal test is about 200 to 300 . Typically, for a given year or breeding cycle, 10 to 15 parents are selected from a pool of 200 parents selected for second-generation breeding and controlled pair matings are performed to produce about 20 to 30 full-sib families. Within each family, about $10 \mathrm{SE}$ clones are developed and planted in the test, while the corresponding ET lines are cryopreserved. At each test site, 6 to 8 ramets per clone should be sufficient to rank clone means. Tests will be monitored periodically until rotation age.

\section{MANAGING DIVERSITY IN CLONAL FORESTRY}

One major concern about deploying clones in plantations is that a narrow genetic base may make clonal plantations more vulnerable to diseases and insects than trees in a natural forest, thus leading to plantation failure. However, in contrast to highly domesticated agricultural crop plants, most forest tree populations have a wide range of genetic variability for pest-resistance characteristics. Therefore, individuals that show a high level of pest resistance can be selected for cloning and deployment in clonal forestry, especially for known pests, and thus more resistant clones may be developed. For unknown insects and diseases, however, the protection is rather limited, despite the high degree of genetic variability.

There is a risk that susceptible genotypes will unknowingly be deployed in the clonal plantation. In general, it is assumed that the more genotypes deployed in a clonal plantation, the lower the risk. However, increasing the number of clones in a plantation will result in a reduction in genetic gain. Thus, a balancing act is required, giving rise to the question "What is a safe number of clones in a clonal plantation?" [8]. This is a difficult question, because the pest-host system is complex and model building is difficult when dealing with totally unknown diseases and insects. However, using various approaches to quantify this question, scientists generally agree that planting $15-30$ clones mixed in a plantation should be sufficient for protection yet still confer the benefits of clonal forestry $[6,8,15-17]$. Here are some general considerations in determining the number of clones that should be used in clonal plantations [10]: (1) if the species is short lived or short rotation, a lower number of clones may be used because the exposure period to potential risk is reduced; (2) a lower number may be acceptable if forest management systems are intensive and include pest control measures and (3) the more well known a clone, the more acceptable is its extensive use.

Once the appropriate number of clones has been decided, a deployment strategy must consider the configuration of deployed clones. Such a configuration can consist of clones in a random mixture or mono-clonal blocks in a mosaic structure [9]. Alternatively, a "mixture of clones and seedlings 
(MOCAS)" is proposed for New Brunswick. For example, in a plantation, $60 \%$ of the plants can be a mixture of the best clones identified from genetic tests and the remaining $40 \%$ of plants can be propagated from low-cost seed orchard seed. Besides reducing the cost of planting stock, as the clonal stock is more expensive, MOCAS will increase the initial diversity of the plantation. Another reason for proposing MOCAS is that, typically, about $40 \%$ of the plantation basal area is commercially thinned by one-half rotation age, leaving superior quality trees for final harvesting. It is likely that the remaining crop trees will be tested clones and the best trees propagated from seed orchard seeds. This will maintain the initial high level of diversity and reduce the time that remaining clones are exposed to potential risk. Clonal forestry would also shorten the rotation age.

As implied, clonal forestry must be based on tree breeding and genetic testing. Through tree breeding, progressively improved trees are produced at each generation. Genetic testing of clones obtained at each progressive breeding cycle will produce further improved clones. Therefore, the composition of clonal mixtures in subsequent clonal plantations will change over time. Furthermore, evaluation of genetic tests at regular intervals until the rotation age will lead to continually revised clonal compositions that are available for each clonal plantation establishment. Thus, the diversity of clonal plantations can also be managed through time.

\section{CONCLUDING REMARKS}

Clonal forestry, the deployment of clonal varieties or tested clones in plantations, offers much greater genetic gain than is possible through conventional tree breeding, as well as unprecedented flexibility to refine breeding and product goals. SE and cryopreservation are the key technologies that make the practice of clonal forestry possible. For $P$. glauca, $P$. mariana, $P$. abies and $P$. strobus breeding programs in eastern Canada, the implementation of clonal forestry using $\mathrm{SE}$ is feasible despite a lack of artificial seed technology. This is because SE induction and subsequent plant conversion rates are high and the alternative clonal multiplication by rooting of cuttings from juvenile donor plants can be accomplished easily. Clonal field tests containing a large number of clones are essential to obtain a large genetic gain. Depending on the degree of genetic gain pursued, the diversity of clonal plantations can be managed by selecting an appropriate number of clones and configuring various clone mixtures.

In the past 20 years, more than 35 ha of clonally propagated genetic tests and demonstration plantings have been established in the Maritimes region of Canada. The earlier clonal plantations have been established using rooted cuttings of P. glauca, P. mariana, Larix laricia, and L. eurolepis. Recently, in the past 7 years, the clonal plantations have been established using SE-derived trees of P. glauca, P. mariana, P. strobus, and P. banksiana. The older plantations of rooted cuttings have demonstrated the feasibility of implementing clonal forestry with these species. The SE-derived plantations are too young to draw conclusive recommendations; however, the prospect of using SE in clonal forestry is encouraging, as SE-derived trees do not show any abnormality to date (as old as age 9). We expect these plantations will provide valuable information as the plantations develop.

\section{REFERENCES}

[1] Cyr D.R., Cryopreservation of embryogenic cultures of conifers and its application to clonal forestry, in: Jain S.M., Gupta P.K., Newton R.J. (Eds.), Somatic Embryogenesis in Woody Plants, Vol. 4, Kluwer Acad. Publ., Dordrecht, The Netherlands, 1999, pp. 239-261.

[2] DeVerno L.L., Park Y.S., Bonga J.M., Barrett J.D., Somaclonal variation in cryopreserved embryogenic clones of white spruce [Picea glauca (Moench) Voss], Plant Cell Rep. 18 (1999) 948-953.

[3] Garin E., Isabel N., Plourde A., Screening of large numbers of seed families of Pinus strobus L. for somatic embryogenesis from immature and mature zygotic embryos, Plant Cell Rep. 18 (1998) 37-43.

[4] Grossnickle S.C., Major J.E., Interior spruce seedlings compared to emblings produced from somatic embryogenesis. II. Stock quality assessment prior to field planting, Can. J. For. Res. 24 (1994) 1385-1396.

[5] Högberg K.-A., Ekberg I., Norell L., von Arnold S., Integration of somatic embryogenesis in a tree breeding programme: a case study with Picea abies, Can. J. For. Res. 28 (1998) 1536-1545.

[6] Hühn M., Clonal mixtures, juvenile-mature correlations and necessary number of clones, Silvae Genet. 36 (1987) 83-92.

[7] Klimaszewska K., Park Y.S., Overton C., MacEacheron I., Bonga J.M., Optimized somatic embryogenesis, In vitro Cell. Dev. Biol. Plant (2001) 231-239.

[8] Libby W.J., What is a safe number of clones per plantation?, in: Heybrook H.M., Stephan B.R., von Weissenberg K. (Eds.), Resistance to disease and pests in forest trees, Pudoc, Wageningen, The Netherlands, 1982, pp. 342-360.

[9] Libby W.J., Testing and deployment of genetically engineered trees, in: Bonga J.M., Durzan D.J. (Eds.), Cell and Tissue Culture in Forestry, Martinus Nijhoff Publ., Dordrecht, The Netherlands, 1987, pp. 167-197.

[10] Lindgren D., The population biology of clonal deloyment, in: Ahuja M.R., Libby W.J. (Eds.), Clonal Forestry I: Genetics and Biotechnology, Springer-Verlag, Berlin, Germany, 1993, pp. 34-49.

[11] Park Y.S., Pond S.E., Bonga J.M., Initiation of somatic embryogenesis in white spruce (Picea glauca): genetic control, culture treatment effects, and implications for tree breeding, Theor. Appl. Genet. 86 (1993) 427-436.

[12] Park Y.S., Pond S.E., Bonga J.M., Somatic embryogenesis in white spruce (Picea glauca): genetic control in somatic embryos exposed to storage, maturation treatments, germination and cryopreservation, Theor. Appl. Genet. 89 (1994) 742-750.

[13] Park Y.S., Barrett J.D., Bonga J.M., Application of somatic embryogenesis in high-value clonal forestry: deployment, genetic control, and stability of cryopreserved clones, In vitro Cell. Dev. Biol. Plant 34 (1998) 231-239.

[14] Park Y.S., Bonga J.M., Mullin T.J., Clonal Forestry, in: Mandal A.K., Gibson G.L. (Eds.), Forest Genetics and Tree Breeding, CBS Publ. \& Distributors, New Delhi, India, 1998, pp. 143-167.

[15] Roberds J.H., Bisher J.W., Risk analyses in clonal forestry, Can. J. For. Res. 27 (1997) 425-432.

[16] Sutton B., Commercial delivery of genetic improvement to conifer plantations using somatic embryogenesis, Ann. For. Sci. 59 (2002) 657-661.

[17] Zobel B., Clonal forestry in the Eucalypts, in: Ahuja M.R., Libby W.J. (Eds.), Clonal Forestry I: Genetics and Biotechnology, Springer-Verlag, Berlin, Germany, 1993, pp. 139-148. 